\title{
Gram-negative infective endocarditis: a retrospective analysis of 10 years data on clinical spectrum, risk factor and outcome
}

\author{
Vineeth Varghese Thomas ${ }^{1}$, Ajay Kumar Mishra ${ }^{2}$, Sudha Jasmine ${ }^{1}$, Sowmya Sathyendra ${ }^{1}$ \\ ${ }^{1}$ Internal Medicine, Christian Medical College and Hospital, Vellore, Tamil Nadu, India; ${ }^{2}$ Internal Medicine, Saint \\ Vincent Hospital, Worcester, MA, USA
}

\begin{abstract}
Infective endocarditis (IE) is a significant cause of morbidity and mortality. Underlying congenital heart disease and acquired valvular disease significantly increases the IE risk, which is still prevalent in developing countries. Gram-negative organism related IE prevalence appears to be rising with limited data on their presentation and outcomes. This study hopes to shed further light on this subject. This retrospective cross-sectional study occurred in a tertiary care center in South India.

A retrospective cross-sectional study performed in a single tertiary care center in South India. All patients with IE from 2006 to 2016 were included in this study. The details of clinical presentation, laboratory investigations, clinical course, microbiology, and outcomes were obtained. Patients fulfilling the modi-
\end{abstract}

Correspondence: Ajay Kumar Mishra, Internal Medicine, Saint Vincent Hospital 123 Summer Street, Worcester, MA, USA.

Tel.: 508-363-500.

E-mail: Mishra@stvincenthospital.com

Key words: Infective endocarditis; bacteria; gram negative; outcome.

Conflict of interest: The authors declare no conflict of interest.

Funding: None.

Ethics approval: IRB clearance obtained.

Consent: Not required.

Contributions: AKM, SJ, SS planned and formulated the study. VVT obtained the IRB clearance. VVT collected and analyzed the data. VVT, AKM completed the manuscript. AKM, SJ, SS reviewed and approved the manuscript.

Received for publication: 5 May 2020.

Accepted for publication: 22 July 2020.

${ }^{\circ}$ Copyright: the Author(s), 2020

Licensee PAGEPress, Italy

Monaldi Archives for Chest Disease 2020; 90:1359

doi: 10.4081/monaldi.2020.1359

This article is distributed under the terms of the Creative Commons Attribution Noncommercial License (by-nc 4.0) which permits any noncommercial use, distribution, and reproduction in any medium, provided the original author(s) and source are credited. fied Duke's criteria and a culture-proven diagnosis of gram-negative IE were eligible for inclusion. A total of 27 patients were enrolled from Jan 2006 to Dec 2016, among whom 78\% were male. Prior structural heart disease was common in our cohort (41\%) with renal (55\%) and embolic (51\%) complications being the most common systemic complications. A comparison of mortality with survivors found that congenital and acquired structural heart disease had a higher risk of mortality. Non-fermenting GNB accounted for $52 \%$ of the cohort, with Pseudomonas accounting for $19 \%$. E. coli was the most common bacilli isolated, constituting $37 \%$ of the cohort. Assessment of risk factors for adverse outcomes found that renal dysfunction and intravascular device were significant with multivariate-logarithmic analysis showing renal dysfunction as an independent risk factor. In-hospital mortality in this series was $30 \%$.

In conclusion, gram-negative IE was more prevalent among males. Underlying structural heart disease was the most common risk factor associated with the disease. Renal dysfunction and embolic complications were the most common complications in this cohort. E. coli and NFGNB accounted for $70 \%$ of the offending organisms. In-hospital mortality was similar to patients with IE secondary to common organisms. The presence of renal dysfunction was an independent risk factor for an adverse outcome.

\section{Introduction}

Infective endocarditis (IE) is an infection of the heart chambers and valves and is associated with significant morbidity and mortality $[1,2]$. It is a conventional differential for patients presenting with pyrexia of unknown origin (PUO) in the background of pre-existing valvular heart disease $[3,4]$. The most common bacterial agents causing IE are gram-positive cocci. Over the last 50 years, Incidences of gram-negative endocarditis is increasing $[5,6]$. However, there is limited data on its characteristics and outcomes. In this retrospective cross-sectional study, we evaluated the patients with gram-negative IE regarding the same.

\section{Aims}

In this study, we aimed to analyze the epidemiological, clinical, laboratory characteristics of patients with gram-negative IE. We also assessed the various risk factors and outcomes among these patients. 


\section{Methodology}

The retrospective cross-sectional study was conducted in a tertiary care health center in South India. The medical records of all patients with a diagnosis of IE were reviewed. Patients admitted from 2006 to 2016 were recruited. The total number of admissions during the same period was 75,719. Among these, 256 patients were diagnosed to have IE. Among these, 27 patients fulfilled the modified Duke's criteria for the diagnosis of IE and had cultureproven isolates of gram-negative organisms. Those who did not fulfill the diagnostic criteria were excluded from the study. Details of demography, clinical features, hematological, biochemical, and microbiological parameters were obtained. The principal investigator tried to ascertain the presence of risk factors, treatment details, and outcomes in each patient. A trained physician did data recruitment, analysis, and interpretation. Data were entered in Microsoft Excel and were analyzed using Stata 13.

\section{Results}

Among the 75,719 patients, 256 patients were identified to have IE $(0.33 \%)$ (Figure 1$)$. Among these, the prevalence of gramnegative endocarditis was $10.7 \%(\mathrm{~N}=27)$. Analysis of the baseline characteristics (Table 1) revealed that the median age of patients was 48.5 years, with the youngest being 18 years and the oldest 75 years. Seventy-eight percent $(\mathrm{N}=21)$ of the patients were male; $40 \%(\mathrm{~N}=11)$ had a prior history of structural heart diseases, including rheumatic valvular heart disease, and valve replacement; $35 \%$ $(\mathrm{N}=10)$ of these patients had a prior history of IE. History of prolonged fever was present in all patients $(100 \%)$, followed by the presence of significant weight loss in $40 \%(\mathrm{~N}=11)$ patients. On clinical examination, new-onset murmur $(\mathrm{N}=16)$ and a palpable spleen $(\mathrm{N}=9)$ were present in $60 \%$ and $33 \%$, respectively.

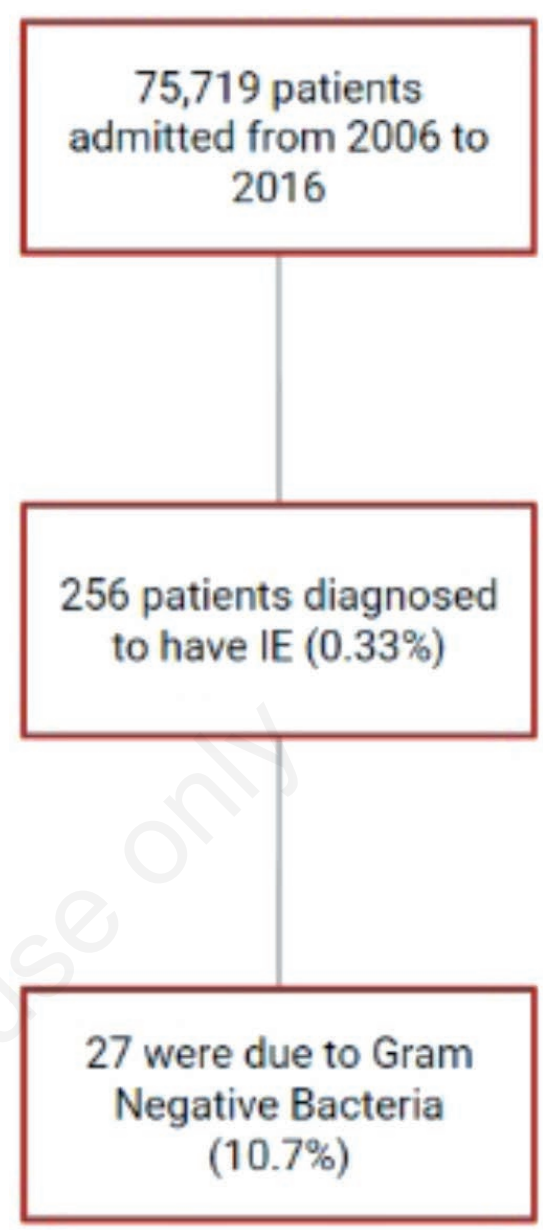

Figure 1. Strobe diagram showing number of patients in the study.

Table 1. Showing demographic information, risk factors, and clinical features and systemic complications.

\begin{tabular}{|c|c|c|c|c|c|}
\hline Variable & Full cohort & Good outcome & Bad outcome & p-Value* & OR $(95 \% \mathrm{CI})$ \\
\hline \multicolumn{6}{|l|}{ Demographic Details } \\
\hline Age (median years, min-max) & $48.5 \mathrm{yrs}(18-75)$ & $47(18-69)$ & $50(24-75)$ & 0.577 & - \\
\hline Gender (Males value, \%) & 21/27 (78\%) & $9(42 \%)$ & $12(57 \%)$ & 1.000 & $0.67(0.1-4.5)$ \\
\hline \multicolumn{6}{|l|}{ Clinical Features (Value, \%) } \\
\hline Fever & $27 / 27(100 \%)$ & $11(100 \%)$ & $16(100 \%)$ & $\underline{ }$ & - \\
\hline Weight Loss & $11 / 27(41 \%)$ & $5(45 \%)$ & $6(55 \%)$ & $\underline{-}$ & $\underline{ }$ \\
\hline New murmur & $16 / 27(60 \%)$ & $8(50 \%)$ & $8(50 \%)$ & - & - \\
\hline Splenomegaly & $9 / 27(33 \%)$ & $6(67 \%)$ & $3(33 \%)$ & $\underline{-}$ & $\underline{-}$ \\
\hline \multicolumn{6}{|l|}{ Risk Factors (Value,\%) } \\
\hline Prior structural heart disease & $11 / 23(41 \%)$ & $4(36 \%)$ & $7(63 \%)$ & 0.292 & $2.5(0.5-13.1)$ \\
\hline Past history of infective endocarditis & $10 / 23(35 \%)$ & $4(36 \%)$ & $6(54 \%)$ & 0.667 & $1.9(0.3-11.0)$ \\
\hline Previous cardiac surgery & $11 / 23(41 \%)$ & $4(36 \%)$ & $7(64 \%)$ & 0.414 & $2.5(0.5-13.2)$ \\
\hline Previous Intravascular device & $7 / 23(25 \%)$ & $4(57 \%)$ & $3(43 \%)$ & 0.045 & $0.6(0.1-3.5)$ \\
\hline \multicolumn{6}{|c|}{ Systemic Complications (Value, \%) } \\
\hline Renal dysfunction & $15 / 27(55 \%)$ & $3(20 \%)$ & $12(80 \%)$ & 0.022 & $8.0(1.4-45.8)$ \\
\hline Embolic phenomenon & $14 / 26(51 \%)$ & $5(36 \%)$ & $9(64 \%)$ & 0.462 & $1.8(0.4-8.7)$ \\
\hline Metastatic infection & 10/26 (37\%) & $3(30 \%)$ & $7(70 \%)$ & 0.448 & $2.1(0.4-10.8)$ \\
\hline Neurological complications & $10 / 26(37 \%)$ & $3(30 \%)$ & $7(70 \%)$ & 0.428 & $2.3(0.4-12.4)$ \\
\hline
\end{tabular}

* p-value: statistical analysis performed against 'bad outcome'. 
All the blood cultures were obtained as per the requirement of Duke's criteria, and all the patients fulfilled the major criteria for blood culture positivity. Analysis of microbiological species showed that $37 \%$ (10) had E. coli with non-pseudomonas NFGNB constituting 33\% (9). Pseudomonas species were grown in 19\% (5), while Salmonella, Citrobacter, and Hemophilus were grown in one each (Table 2). The incubation period (time to reporting of cultures) was less than 48 hours in $25 \%$ (7) of the patients. Of the remaining 21 cases, $70 \%$ (16) took 2 to 7 days, while 30\% (5) took more than a week to grow. The organisms' antibiotic sensitivity profile revealed sensitivity to 3rd generation Cephalosporins in $45 \%$ (12) of the cases; $41 \%$ (11) cases were resistant to cefoperazone, while $11 \%$ (3) were resistant to carbapenems.

On evaluating risk factors, it was found that the prevalence of underlying structural heart disease was present in $40 \%$ of our cases with rheumatic heart disease accounting for nearly all of them. $35 \%$ of our cohort also had previously been diagnosed and treated for IE. Almost $40 \%$ of the group had a prior history of cardiac surgery commonly for valve replacement due to severe stenotic lesions widely seen in rheumatic heart disease. Among these patient's analyses of complications showed that renal dysfunction was present in $60 \%$ (16) of the cases, while $52 \%$ (14) had an embolic phenomenon. Among the embolic event, metastatic infection and neurological complications like stroke or TIA were noted in $37 \%$ (10) cases. The other complications reported were mesenteric ischemia and peripheral end artery occlusion. Analysis of risk factors of our cohort with in-hospital mortality revealed that previous intravascular device and the presence of renal dysfunction was associated with higher mortality (Table 2). The in-hospital mortality was found to be $30 \%$ (Figure 2 ).

Analysis of the antibiotic sensitivity profile was obtained from the records (Table 2). A large proportion of Pseudomonas (60\%) and NFGNB (44\%) were ESBL, while $30 \%$ of E. coli were in the same category. Aminoglycoside and fluoroquinolone susceptibility pattern for Pseudomonas was above $80 \%$. However, it was below $50 \%$ for $E$. coli and NF-GNB. Sensitivity patterns towards colistin were $100 \%$ in all the isolates. The culture with Salmonella, Citrobacter and Hemophilus were sensitive to all three classes of antibiotics.

A comparison between the various risk factors, complications, and microbiological parameters was done, between the survivors and the nonsurvivors (Table 3). Congenital and structural heart disease was present in $50 \%$ and $100 \%$ of the deaths, respectively, compared to none in the survivors. Analysis of etiological agents revealed that NF-GNB and $E$. coli were associated with the highest mortality (44\%) while Pseudomonas and other organisms had done well with therapy.

As some of the patients had been discharged in an unstable clinical condition with the risk of mortality, they were analyzed with in-hospital mortality groups as "adverse-outcome" against the remaining survivors (Table 4). Microbiological analysis of ESBL vs. non-ESBL infection was not significant.

\section{Discussion}

Our study analyzed the risk factors commonly associated with IE. We also determined the complications and outcomes in patients admitted with gram-negative IE. Studies on IE have been scanty from the developing countries. Most data available on IE are from developed countries [7]. Subacute IE is much more prevalent in the Indian subcontinent as compared to the western population due to the higher prevalence of rheumatic heart disease [8]. Western pop-

Table 2. Antibiotic sensitivity profile.

\begin{tabular}{|c|c|c|c|c|}
\hline & E. coli (10) & NF-GNB (9) & Pseudomonas (5) & Others (3) \\
\hline Aminoglycosides & $42 \%$ & $34 \%$ & $80 \%$ & $100 \%$ \\
\hline Cefpodoxime & $30 \%$ & $44 \%$ & $60 \%$ & $100 \%$ \\
\hline Fluoroquinolones & $30 \%$ & $44 \%$ & $80 \%$ & $100 \%$ \\
\hline Colistin & $100 \%$ & $90 \%$ & $100 \%$ & 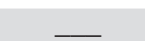 \\
\hline
\end{tabular}

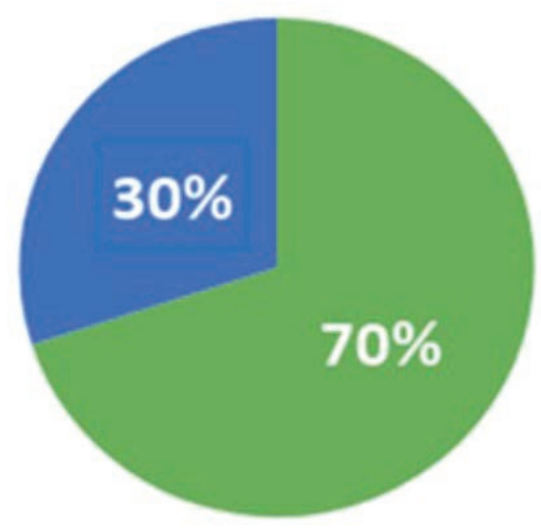

Discharged Deceased

Figure 2. Showing outcome as in-hospital mortality.
Table 3. Comparison of risk factors between survivors and mortality. Variable Survivors (\%) Mortality (\%)

\section{Risk factors (\%)}

Congenital heart disease

$\begin{array}{lll}\text { Previous infective endocarditis } & 50 \% & 92 \%\end{array}$

Complications (\%)

Embolic phenomenon

Metastatic infection

Neurological complication

$0 \%$

Microbiology (value, \%)

Escherichia coli

Pseudomonas

Non-fermenting gram-negative bacteria Others

$7(58 \%)$
$3(100 \%)$ 
ulation-based studies report an incidence of fewer than 10 cases for 100,000 years [9]. In our study, we found that IE accounted for 1 in 300 in hospital admission. IE in our study was commonly seen in patients being evaluated for Pyrexia of Unknown Origin (PUO).

Gram-positive organisms account for more than $70 \%$ of cases of infective endocarditis and are the primary etiological agent for community-acquired and hospital-acquired cases of IE [10]. Common gram-positive organisms implicated are staphylococcus, streptococcus, and enterococcus. Aerobic gram-negative organisms, which are a common cause for bloodstream infections, do not usually cause IE [11]. These commonly present as acute febrile illness progressing to sepsis. Gram-negative organisms account for less than $5 \%$ of the IE. The organisms commonly implicated are $E$. coli, Klebsiella sp, Enterobacteriaceae and Pseudomonas species [11]. In our study, gram-negative endocarditis accounted for $10.6 \%$ of the cases. There are no population-based studies from India regarding the prevalence of gram-negative organism related IE.

The median age of patients with IE was 25 years, given the higher prevalence of rheumatic heart disease [8]. Although early studies had shown that males had a higher prevalence of IE, recent studies have shown a change in trend with equal distribution between the sexes [9]. In our study, the median age was 49 years, with a male preponderance of $78 \%$. This difference could be attributed to patterns of health-seeking behavior in our community and recruitment bias. The presence of prior structural cardiac lesions accounts for $40 \%-70 \%$ of cases with IE $[12,13]$. Health care exposure has been identified as a novel risk factor in addition to the traditional risk factors of underlying structural heart lesions (congenital or acquired). Patients having indwelling catheters, invasive lines, intracardiac devices are at a higher risk for IE. Among these patients, the risk of drug-resistant organisms, complications, and mortality tends to be higher [14]. In our study, the common predisposing factors were underlying structural heart disease (rheumatic heart disease), previous infective endocarditis, and a prior history of cardiac surgery. Intravascular lines or devices which can act as a nidus for infection can also predispose to IE secondary to gramnegative organisms [15]. One of the patients in the cohort had an infected ICD lead.

Fever has been the most common clinical feature at the presentation of IE, similar to our study [16]. The incidence of newonset murmur was found to be $48 \%$, and weight loss was seen in $30 \%$ of patients in one large prospective study [16]. The prevalence of new-onset murmur and weight loss in our study was $60 \%$ and $40 \%$, respectively. While the previous study reported splenomegaly in $11 \%$ of patients, $33 \%$ of our patients had presented with splenomegaly [17].
In our study, $60 \%$ of patients had renal dysfunction, either defined as an elevated creatinine or presence of casts suggestive of an underlying systemic immune disorder. The literature on the prevalence of renal involvement in patients with IE is scanty. However, in this study, renal dysfunction is shown to be associated with poor outcomes. Embolic complications can be subdivided into those with and without involving the central nervous system. Neurological events, including stroke, were reported in $17 \%$ to $40 \%$ of patients. While non-stroke embolization accounts for $23 \%$ of the cohort $[18,19]$. Septic embolization involving visceral organs, pulmonary vasculature, bones, and joints have been associated with high morbidity and mortality. The extent and outcome of embolization are dependent on early diagnosis and initiation of treatment, especially in patients with native valve endocarditis [20]. In our study, the evidence of systemic embolization was found in $52 \%$, with $37 \%$ of these presenting with neurological complications like transient ischemic attacks or stroke. Metastatic infections were observed in $37 \%$ of our patients, with the involvement of visceral organs and osteomyelitis being the more common. There was one case of mesenteric ischemia, which subsequently led to infarction.

Gram-positive organisms account for more than $70 \%$ of cases of IE and are the primary etiological agent for communityacquired and hospital-acquired cases of IE. The species commonly included are staphylococcus, streptococcus, and enterococcus. Gram-negative organisms account for less than $5 \%$ of the total incidence of IE $[16,21]$. In a previous case series of 56 patients with gram-negative endocarditis, HACEK organisms accounted for $100 \%$ of the cases [22]. In our study, nearly $50 \%$ of cultures had grown non-fermenting GNB, of which $20 \%$ were speciated to be due to Pseudomonas. $38 \%$ of our cultures had grown E. coli while there were isolated cases of Salmonella, Citrobacter and Hemophilus. It was noted that $78 \%$ of the cultures required a minimum incubation period of 48 hours to identify the organism, while $20 \%$ required an incubation period of more than a week. Sensitivity profile data revealed only $45 \%$ were sensitive to cephalosporins, while carbapenems or colistin were required in the remaining cases. As compared to IE secondary to the gram-positive organism, gram-negative IE is associated with similar morbidity and mortality [16,22-24]. Our study found an in-hospital mortality rate of $30 \%$ similar to published literature [25].

Statistical analysis of risk factors and complications of IE with mortality revealed that renal dysfunction and the presence of intravascular devices were associated with a higher risk of mortality $[26,27]$. These factors were put-through multivariate logarithmic regression analysis, which showed that renal dysfunction pos-

Table 4. Risk factors associated with a bad outcome.

\begin{tabular}{|c|c|c|c|c|c|}
\hline Variable & Value $(\%)$ & p-Value & Odds Ratio (95\% CI) & Log-regression analysis & Adjusted OR (95\% CI) \\
\hline ESBL Microorganism Infection & $15 / 27(55)$ & 0.130 & $3.8(0.7-19.5)$ & N/A & N/A \\
\hline \multicolumn{6}{|l|}{ Risk factors } \\
\hline Renal Dysfunction & $12 / 16(75)$ & 0.022 & $8.0(1.4-45.8)$ & 0.038 & $0.068(0.005-0.860)$ \\
\hline Use of an intravascular device & $11 / 15(72)$ & 0.020 & $7.3(1.3-42.3)$ & 0.481 & $0.432(0.042-4.473)$ \\
\hline ICU Admission & $6 / 16(22)$ & 0.054 & N/A & N/A & N/A \\
\hline \multicolumn{6}{|l|}{ Investigations: } \\
\hline Coagulopathy & $5 / 13(40)$ & 0.041 & N/A & 0.999 & N/A \\
\hline Albumin less than $3.0 \mathrm{mg} / \mathrm{dl}$ & $9 / 15(60)$ & 1.00 & $1.2(0.2-6.4)$ & N/A & $\mathrm{N} / \mathrm{A}$ \\
\hline Creatinine more than $1.4 \mathrm{mg} / \mathrm{dl}$ & 11/14 (78) & 0.54 & N/A & $\mathrm{N} / \mathrm{A}$ & $\mathrm{N} / \mathrm{A}$ \\
\hline
\end{tabular}


itively correlated with adverse-outcome. Our study had several limitations; we did not have details of cardiac imaging, transesophageal echocardiogram, details of surgical management, details of recurrence, and long term outcomes on these patients [28-30]. The strength of our study was the sample size, longer duration of the study, availability of microbiological diagnosis, and antibiotics susceptibility pattern for all patients [31-33].

In view of high mortality (30\%), IE continues to be a feared disease across the developed world. More than half of the patients have a normal heart at diagnosis, and the proportion of healthcare-related IE is on the rise [33]. With the increasing longevity of the population, coupled with the utilization of invasive procedures, indwelling devices, catheters in the management of patients, the prevalence of IE is on the rise $[33,34]$. Healthcare-related IE contributes to onethird of patients with IE in high-income countries [35]. A large proportion of gram-negative endocarditis in our cohort is also primarily as a result of healthcare-related events. Furthermore, the microbiological sensitivity patterns reveal that drug resistance is becoming a problem contributing to patient morbidity and the cost of treatment $[36,37]$. Epidemiology, microbiological profile, drug susceptibility, adverse drug effects, and management guidelines on IE are continually changing [35,38-40]. The present study should serve as a marker to increase our vigilance towards IE secondary to gram-negative organisms, and promote further studies in this area.

\section{Conclusions}

Gram-negative IE was prevalent more among the male population, with underlying structural heart disease. Renal dysfunction and embolic complications are the most common complications in this subgroup of patients. E. coli and NFGNB account for $70 \%$ of the offending organisms. Reported in-hospital mortality is $30 \%$. Presence of renal dysfunction is an independent risk factor for adverse outcome

\section{References}

1. Mishra AK, Sahu KK, Lal A, Menon V. Aortic valve abscess: Staphylococcus epidermidis and infective endocarditis, QJM 2020;113:211-2.

2. Sahu KK, Sherif AA, Syed MP, et al. A rare cause of sepsis: Lactococcus garvieae. QJM 2019;112:447-8. doi:10.1093/ qjmed/hcz078.

3. Mishra AK, Hashmath Z, Oneyssi I, Bose A. Disseminated Erythema Migrans. Am J Med 2020;133:e374-e375. doi: 10.1016/j.amjmed.2019.12.051.

4. Mishra AK, George AA, Sadhasiv M, Sathyendra S. 'Chik' Sign in Chikungunya Fever. J Assoc Physicians India 2016;64:74.

5. Sahu KK, Tsitsilianos N, Moselle L, Mishra AK. Septic arthritis of hip joint and its devastating complications. BMJ Case Rep 2020;13:e233909. doi:10.1136/bcr-2019-233909.

6. Mishra AK, Lahiri A. Aspergillosis following bronchial artery embolization. QJM 2020;hcaa115. doi:10.1093/qjmed/hcaa115.

7. Tleyjeh IM, Abdel-Latif A, Rahbi H, et al. A systematic review of population-based studies of infective endocarditis. Chest 2007; 132:1025-35.

8. Choudhury R, Grover A, Varma J, et al. Active infective endocarditis observed in an Indian hospital 1981-1991. Am J Cardiol 1992;70:1453-8.
9. Correa de Sa DD, Tleyjeh IM, Anavekar NS, et al. Epidemiological trends of infective endocarditis: a populationbased study in Olmsted County, Minnesota. Mayo Clin Proc 2010;85:422-6.

10. Mishra AK, Sahu KK, Baddam V, Sargent J. Stroke and infective endocarditis. QJM 2020;113:515-6. doi: 10.1093/qjmed/ hcaa098.

11. Raza SS, Sultan OW, Sohail MR. Gram-negative bacterial endocarditis in adults: state-of-the-heart. Expert Rev Anti Infect Ther 2010;8:879-85.

12. Michel PL, Acar J. Native cardiac disease predisposing to infective endocarditis. Eur Heart J 1995;16:2-6.

13. Mishra AK, Sahu KK, George AA, Lal A. Safety and efficacy of thrombolysis and mechanical thrombectomy in infective endocarditis. J Stroke Cerebrovasc Dis 2020:104784. doi: 10.1016/j.jstrokecerebrovasdis.2020.104784.

14. Siegman-Igra Y, Koifman B, Porat R, et al. Healthcare associated infective endocarditis: a distinct entity. Scand J Infect Dis 2008;40:474-80.

15. Carrel T, Schaffner A, Vogt P, et al. Endocarditis in intravenous drug addicts and HIV infected patients: possibilities and limitations of surgical treatment. J Heart Valve Dis 1993;2:140-7.

16. Murdoch DR, Corey GR, Hoen B, et al. Clinical presentation, etiology, and outcome of infective endocarditis in the 21 st century: the International Collaboration on EndocarditisProspective Cohort Study. Arch Intern Med 2009;169:463-73.

17. Durante-Mangoni E, Bradley S, Selton-Suty C, et al. Current features of infective endocarditis in elderly patients: results of the International Collaboration on Endocarditis Prospective Cohort Study. Arch Intern Med 2008;168:2095-103.

18. Mishra AK, Sahu KK, Lal A, Sujata M. Systemic embolization following fungal infective endocarditis, QJM 2020;113:233-35.

19. Baddour LM, Wilson WR, Bayer AS, et al. Infective endocarditis: diagnosis, antimicrobial therapy, and management of complications: a statement for healthcare professionals from the Committee on Rheumatic Fever, Endocarditis, and Kawasaki Disease, Council on Cardiovascular Disease in the Young, and the Councils on Clinical Cardiology, Stroke, and Cardiovascular Surgery and Anesthesia, American Heart Association: endorsed by the Infectious Diseases Society of America. Circulation 2005; 111:e394-434.

20. Habib G, Hoen B, Tornos P, et al. Guidelines on the prevention, diagnosis, and treatment of infective endocarditis (new version 2009): the Task Force on the Prevention, Diagnosis, and Treatment of Infective Endocarditis of the European Society of Cardiology (ESC). Endorsed by the European Society of Clinical Microbiology and Infectious Diseases (ESCMID) and the International Society of Chemotherapy (ISC) for Infection and Cancer. Eur Heart J 2009;30:2369-413.

21. Selton-Suty C, Célard M, Le Moing V, et al. Preeminence of Staphylococcus aureus in infective endocarditis: a 1-year population-based survey. Clin Infect Dis Off Publ Infect Dis Soc Am 2012;54:1230-9.

22. Geraci JE, Wilson WR. Symposium on infective endocarditis. III. Endocarditis due to gram-negative bacteria. Report of 56 cases. Mayo Clin Proc 1982;57:145-8.

23. Mishra AK, Sahu KK, Lal A, Menon V. Aortic valve abscess: Staphylococcus epidermidis and infective endocarditis, QJM 2020;113:211-2.

24. Ninan F, Mishra AK, John AO, Iyadurai R. Splenic granuloma: Melioidosis or Tuberculosis? J Family Med Prim Care 2018;7:271-3.

25. Sahu KK, Mishra AK, Lal A, Kranis M. An interesting case of 
expressive aphasia: Enterococcus faecalis-related infective endocarditis complicating as septic emboli, QJM 2020;113;146-7.

26. Heiro M, Nikoskelainen J, Engblom E et al. Neurologic manifestations of infective endocarditis: a 17-year experience in a teaching hospital in Finland. Arch Intern Med 2000;160:2781-7.

27. Mishra AK, Sahu KK, Nagabandi S, Benotti J. Infective endocarditis with mitral leaflet perforation and multiple embolic infarcts, QJM 2020:hcaa026. doi.org/10.1093/qjmed/hcaa026.

28. McKinsey DS, Ratts TE, Bisno AL. Underlying cardiac lesions in adults with infective endocarditis. The changing spectrum. Am J Med 1987;82:681-8.

29. Sahu KK, Doshi A, Mishra AK, et al. A female with five chambers. Neth Heart J 2020:28:174-5.

30. Sahu KK, Mishra AK, Sherif AA, et al. An interesting case of pacemaker endocarditis. Neth Heart J 2019:27:585-6.

31. Abhilash K, Patole S, Jambugulam M, et al. Changing Trends of Infective Endocarditis in India: A South Indian Experience. J Cardiovasc Dis Res 2017;8:56-60.

32. Mishra AK, Aaron S, Abhilash K, et al. Simple telephone call a feasible, useful, and acceptable method of following up patients with cerebrovascular accidents: prospective cohort study in South India. Int J Stroke Off J Int Stroke Soc 2016;11:NP87-8.

33. Cahill TJ, Baddour LM, Habib G, et al. Challenges in Infective Endocarditis. J Am Coll Cardiol 2017;69:325-44. doi:10.1016/ j.jacc.2016.10.066.
34. Lal A, Akhtar J, Isaac S, et al. Unusual cause of chest pain, Bornholm disease, a forgotten entity; case report and review of literature. Respir Med Case Rep 2018;25:270-3. doi:10.1016/ j.rmcr.2018.10.005.

35. Wang A, Gaca JG, Chu VH. Management Considerations in Infective Endocarditis: A Review. JAMA 2018;320:72-83. doi:10.1001/jama.2018.7596.

36. Mishra AK, Sahu KK, Lal A. Significance of prolonged PR interval in infections. QJM 2020;113:150-1. doi:10.1093/ qjmed/hcz192.

37. Mani SSR, Gunasekaran K, Iyyadurai R, et al. Clinical spectrum, susceptibility profile, treatment and outcome of cultureconfirmed brucellosis from South India. Indian J Med Microbiol 2018;36:289-92. doi:10.4103/ijmm.IJMM_18_236.

38. Sadanshiv M, George AA, Mishra AK, Kuriakose CK. Rifampicin-induced immune allergic reaction. Trop Doct 2018;48:156-159. doi:10.1177/0049475517724689.

39. Vincent LL, Otto CM. Infective Endocarditis: Update on Epidemiology, Outcomes, and Management. Curr Cardiol Rep 2018;20:86. doi:10.1007/s11886-018-1043-2.

40. Mishra AK, Sahu KK, George AA, Lal A. Safety and Efficacy of Thrombolysis and Mechanical Thrombectomy in Infective Endocarditis. J Stroke Cerebrovasc Dis 2020;29:104784. doi:10.1016/j.jstrokecerebrovasdis.2020.104784. 